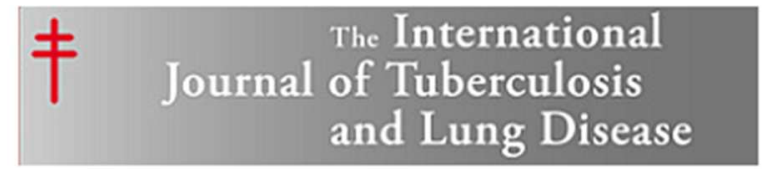

\title{
Tuberculosis in HIV-infected South African children with complicated severe acute malnutrition
}

\begin{tabular}{|c|c|}
\hline Journal: & The International Journal of Tuberculosis and Lung Disease \\
\hline Manuscript ID & IJTLD-10-16-0753.R1 \\
\hline Manuscript Type: & Original Article \\
\hline Date Submitted by the Author: & 12-Nov-2016 \\
\hline Complete List of Authors: & $\begin{array}{l}\text { Adler, Hugh; Mater Misericordiae University Hospital, Infectious Diseases; } \\
\text { King Edward VIII Hospital, Paediatric Infectious Diseases } \\
\text { Archary, Moherndran; King Edward VIII Hospital, Paediatric Infectious } \\
\text { Diseases; University of Kwazulu Natal, Paediatric and Child Health } \\
\text { Mahabeer, Prasha; King Edward VIII Hospital, Microbiology; University of } \\
\text { Kwazulu Natal, Paediatric and Child Health } \\
\text { LaRussa, Philip; Columbia University Medical Center, Pediatric Infectious } \\
\text { Diseases } \\
\text { Bobat, Raziya; King Edward VIII Hospital, Paediatric Infectious Diseases; } \\
\text { University of Kwazulu Natal, Paediatric and Child Health }\end{array}$ \\
\hline Key Words: & kwashiorkor, Protein-Energy Malnutrition, sputum \\
\hline
\end{tabular}


Tuberculosis in HIV-infected South African children with complicated severe acute malnutrition

Running head: TB in children with HIV \& severe malnutrition

Author information

1. Dr Hugh Adler MB BCh BAO, MRCPI, DTM\&H. Dept of Infectious Diseases, Mater Misericordiae University Hospital, Dublin, Ireland; Paediatric Infectious diseases Unit - King Edward VIII Hospital, Durban, South Africa. Current Affiliation: Dept of Clinical Sciences, Liverpool School of Tropical Medicine, Pembroke Place, Liverpool L35QA, UK. Email hugh.adler@gmail.com. Mobile +447490392877

2. Dr Moherndran Archary MBChB, DCH(SA), FCPaeds(SA), Paeds ID (SA). Paediatric Infectious diseases Unit - King Edward VIII Hospital, Durban, South Africa; University of KwaZulu Natal, Durban, South Africa.Email Archary@ukzn.ac.za

3. Dr Prasha Mahabeer MBChB. Microbiology Unit, King Edward VIII Hospital, Durban, South Africa; University of KwaZulu Natal, Durban, South Africa. Email Mahabeerp2@ukzn.ac.za

4. Prof Philip LaRussa, M.D. Division of Pediatric Infectious Diseases, Columbia University Medical Center, New York, NY 10032 USA. Email psl1@cumc.columbia.edu

5. Prof Razia A Bobat MBChB, FCPaeds(SA), MD. Paediatric Infectious diseases Unit King Edward VIII Hospital, Durban, South Africa; University of KwaZulu Natal, Durban, South Africa. Email BOBAT@ukzn.ac.za. Phone +27312604355 (corresponding author)

Word Count of article main text: 2716 words

Key Words: Kwashiorkor, Protein-Energy Malnutrition, Sputum

\section{Contributions:}

All authors had a role in the design of this study. $\mathrm{H}$ Adler, M Archary and P Mahabeer collected the data. H Adler performed statistical analyses and wrote the first draft of the paper, which was revised with contributions from all authors. All authors have approved the final version

Funding and disclosures:

The MATCH study was supported by grant number R24TW008863 from the USA Office of the Global AIDS Coordinator and the Department of Health and Human Services, National Institutes of Health. The contents of this paper are solely the responsibility of the authors and do not necessarily represent the official views of the government. Dr Adler reports receiving travel support from ViiV Healthcare, Janssen pharmaceuticals and Bristol Meyers Squibb. All other authors have no disclosures. 
38 Setting: Academic tertiary referral hospital in Durban, South Africa

39 Objective: To describe the incidence and diagnostic challenges of TB in HIV-infected children 40 with severe acute malnutrition (SAM)

41 Design: Post-hoc analysis of a randomised controlled trial that enrolled ART-naïve, HIV42 infected children with SAM. Trial records and hospital laboratory results were explored for 43 clinical diagnoses and bacteriologically confirmed cases of TB. Negative binomial regression 44 was used to explore associations with confirmed cases of TB, excluding cases where the 45 clinical diagnosis was not supported by microbiological confirmation.

46 Results: Of 82 children enrolled in the study, 21 (25.6\%) were diagnosed with TB, with 47 bacteriological confirmation in 8 cases. Sputum sampling (as opposed to gastric washings) was associated with an increased risk of subsequent diagnosis of TB (adjusted relative risk $1.134,95 \% \mathrm{Cl} 2.1 \%-26 \%)$. A culture-proven bacterial infection during the admission was associated with a reduced risk of TB (aRR $0.856,0.748-0.979)$, which may reflect false negative microbiologic tests secondary to empiric broad spectrum antibiotics.

52 Conclusion: TB is common in HIV-infected children with SAM. While microbiological 53 confirmation of the diagnosis is feasible, empiric treatment remains common, possibly 54 influenced by suboptimal testing and false negative TB diagnostics. Rigorous microbiological 55 TB investigation should be integrated into the programmatic management of HIV and SAM. 
Tuberculosis (TB), HIV infection and severe acute malnutrition (SAM) are individually responsible for high levels of morbidity and mortality among children across sub-Saharan Africa. Research is ongoing into how these three interact, although the potency of the combination has been recognised for some time. ${ }^{1}$

Over 650,000 cases of paediatric TB occur annually in the 22 highest-burden countries, ${ }^{2}$ resulting in an estimated 140,000 annual deaths. ${ }^{3}$ Over 2.6 million children across the world are living with HIV, with $90 \%$ of these living in sub-Saharan Africa. ${ }^{4}$ HIV was responsible for 150,000 childhood deaths in 2014. SAM-incorporating marasmus, kwashiorkor and

In South Africa, over 36,000 cases of childhood TB occur annually, one quarter of which are believed to be HIV co-infected. ${ }^{7,8}$ HIV is responsible for $17 \%$ of all deaths in South African marasmic kwashiorkor-is responsible for as much as $10 \%$ of all global mortality in children aged $<5$ years, ${ }^{5}$ with marasmus alone accounting for over 500,000 deaths annually in this age group. ${ }^{6}$ children aged under 5 years. ${ }^{8}$ One study found that $10 \%$ of children initiating antiretroviral therapy (ART) in rural South Africa also had SAM. ${ }^{9}$

While SAM combined with HIV has already been recognised as a challenging clinical entity, associated with increased mortality, ${ }^{10,11}$ the contribution of TB in this scenario is uncertain. Malnutrition is associated with increased mortality in paediatric TB, ${ }^{12}$ particularly with HIV co-infection ${ }^{13}$. TB causes cachexia and wasting, and may itself be impacted by poor nutritional status. ${ }^{14,15}$ HIV is an independent risk factor for both TB and malnutrition, worsening the outcomes of either condition. ${ }^{10,16}$ The spectrum of TB in a cohort of paediatric patients who all have the specific combination of both HIV and SAM has not yet been studied. A randomised controlled trial (Malnutrition and Antiretroviral Timing in Children with HIV, MATCH) assessing the clinical and pharmacokinetic responses to early versus delayed ART in HIV-infected children with SAM was conducted in Durban, South Africa. ${ }^{17}$ We present a post-hoc analysis of TB diagnoses in these patients. 
$87 \quad$ Setting

King Edward VIII Hospital (KEH) in Durban, South Africa has a 100-bed paediatric ward, including 10 nutritional rehabilitation beds, providing regional tertiary-level care.

\section{Recruitment and eligibility}

All children presenting with SAM and newly diagnosed with HIV were eligible for inclusion in the trial, and randomised either to early (within ten days of admission) or delayed (at four weeks or at protocol-defined nutritional recovery) ART. ${ }^{17}$ Recruitment was carried out between 2012 and 2014. All children eligible for inclusion in the MATCH trial were eligible for inclusion in this retrospective analysis.

SAM was defined as weight-for-height $\mathrm{z}$ score below -3 standard deviations from the World Health Organisation (WHO) median or a mid-upper-arm circumference of $<115 \mathrm{~mm}$, with or without bilateral oedema ${ }^{18}$ and was managed according to the latest WHO guidelines. ${ }^{19}$

Patients were classified as bacteriologically confirmed TB (based on positive sputum smear microscopy, mycobacterial culture or nucleic acid amplification), clinically diagnosed (empirically treated) TB, or no TB, as per the WHO. ${ }^{20}$ A more detailed classification of clinically-diagnosed cases was not possible, as patients' original files and radiographs were frequently unavailable.

Data collection

We retrospectively searched the National Health Laboratory Service's laboratory information management system for TB diagnostic test results from all patients in the MATCH trial, from the date of admission with SAM to any hospital in Durban, through to one month after transfer to $\mathrm{KEH}$ and enrolment in the study. We also recorded routine biochemical, haematological and immunological laboratory results from the day of admission. Clinical parameters and tuberculin skin test results at admission were not captured routinely, as children were only enrolled after admission and a positive HIV test. 
113 As per the local standard of care, investigations for pulmonary TB were performed on at least three samples of induced sputum or gastric washings, at the discretion of the treating clinician. Patients were also investigated for other concurrent infections according to local standard protocols. ${ }^{21}$ Fluorescent microscopy was performed on sputum smears, followed by culture in mycobacterial growth indicator tubes. Specimens that were smear positive and cultures that flagged positive were further subjected to polymerase chain reaction by line-probe assay (LPA; MTBDRplus, Hain Lifescience, Germany). In-vitro drug sensitivity testing was performed for both first and second line anti-tuberculosis drugs. The Xpert MTBRIF platform (hereafter referred to as Xpert) was introduced in late 2013, replacing sputum smear microscopy.

Statistical analysis

We present a descriptive analysis of all TB diagnoses in the MATCH study. TB-negative patients, bacteriologically-confirmed TB cases and clinically-diagnosed cases were compared using ANOVA (for continuously-distributed data) or chi-square (for categorical variables). In addition, we assessed associations between patient characteristics (including laboratory results, TB sampling method and ART strategy) and the diagnosis of TB using a negative binomial logistic regression model comparing children with and without a confirmed diagnosis of TB; empirically-treated children were excluded from this model, as were any children with missing data. We hypothesised that anaemia $(\mathrm{Hb}<10 \mathrm{~g} / \mathrm{dL})$, leukocytosis (white cell count $>12 \times 10^{9}$ ), thrombocytosis (platelets $>400 \times 10^{9}$ ) and hypoalbuminaemia $(<25 \mathrm{~g} / \mathrm{L})$ might, as surrogate markers of inflammation, be associated with a diagnosis of TB (ESR and CRP levels were infrequently measured, and therefore were not included in the model). Following univariate analysis, all variables were included in a fully-adjusted multivariate model. A parsimonious adjusted model was then reached via a backward stepwise approach, sequentially excluding variables whose $p$ value was $>0.1$ and selecting a final model that minimised the Akaike information criterion. Results are presented as adjusted relative risks (aRR) with 95\% confidence intervals $(\mathrm{Cl})$, and $p$ values $<0.05$ were considered significant. All analyses were performed using SPSS version 22 (IBM). 
144 Ethics

145 Written informed consent was obtained from the caregivers of all children enrolled in the

146 MATCH study. The trial was approved by the Biomedical Research Ethics Committee of the 147 University of KwaZulu Natal and King Edward VIII Hospital.

148

149 
RESULTS

\section{Descriptive analysis}

We enrolled 82 children in the MATCH trial. Figure 1 outlines the recruitment and TB diagnostic process, while Table 1 outlines the patients' baseline characteristics and sites of TB. The mean age at admission was 23 months, $46.3 \%(n=38)$ were girls, the mean CD4 percentage was $17.46 \%$ and $21.95 \%(n=18)$ presented with oedematous malnutrition.

Seventy five children were investigated for TB and 21/82 (25.6\%) were determined to have TB. Eight (10.9\%) had bacteriologically confirmed pulmonary TB (one of whom died before the culture flagged positive) while thirteen were clinically diagnosed. Eight were treated empirically after admission, while five were on treatment at enrolment but had no positive TB results on record. All six culture-positive TB isolates were fully sensitive in vitro.

62 patients had sputum samples, while the remaining 13 had gastric aspirates. The mean ages of those with sputum or gastric aspirate samples did not differ significantly ( 25 versus 21 months, $p=0.7)$. No gastric aspirate samples tested positive for TB; two empiricallytreated patients had had gastric aspirate samples tested. Xpert was employed in 45 patients, but was only positive in two, both of whom were subsequently culture positive. Of the 37 patients enrolled before Xpert was widely available, five had confirmed TB and seven were empirically treated, versus three confirmed and six empirically treated after Xpert was introduced $(p=0.199)$. Thus, the introduction of Xpert did not appear to affect the rates of empiric treatment for TB in smear-negative patients, though it should be underlined that this study was carried out while local clinicians were still familiarising themselves with Xpert.

One empirically-treated child was suspected of having TB meningitis clinically and on cerebrospinal fluid analysis (leukocytosis and elevated protein levels), but was TB culture negative from all specimens. The remainder of those commenced on empiric treatment at our centre were suspected of having pulmonary TB. Prior to their admission to KEH, two children were on treatment for suspected pulmonary TB, two for suspected disseminated TB, and one for suspected abdominal TB. In addition, one child underwent an axillary lymph node biopsy which led to a diagnosis of Bacille Calmette-Guérin (BCG) IRIS; this child was not classified as having TB in this study. 


\section{$179 \quad$ Regression analysis}

180 Fifty-three children had sufficient data for inclusion in the multivariate analysis, including 7

181 of those with proven TB (the eighth child, who was smear-positive but culture-negative, was

182 excluded for lack of a full blood count result). Results of the regression analysis are

183 presented in Table 2. While leukocytosis was associated with a slight increased risk of TB in

184 the final model (aRR 1.81, 95\% Cl 1.067-1.308), thrombocytosis was unexpectedly

185 associated with a reduced risk (aRR $0.868,95 \% \mathrm{Cl} 0.774-0.974$ ). The diagnosis of a culture-

186 proven bacterial infection during the admission was associated with a reduced risk of TB

187 (aRR 0.856, 0.748-0.979). Sputum sampling (as opposed to gastric washings) was

188 associated with a $13.4 \%$ increased risk of subsequent diagnosis of TB (95\% $\mathrm{Cl} 2.1 \%-26 \%)$.

189 While allocation to the "delayed ART" group was associated with a $13.8 \%$ increased risk of

190 TB in the full model, this association disappeared when the parsimonious model was

191 created and it was omitted from the final model. There was a small reduction in risk for

192 children with CD4 percentages between 20-25\% (aRR 0.864, 95\% Cl 0.765-0.976 with CD4

193 percentage $>25 \%$ used as the reference category), but no other CD4 values were associated

194 with either increased or decreased risk. 
198 In our prospective cohort of 82 HIV-infected South African children, admitted to a university teaching hospital for the management of complicated SAM, we found a $25.6 \%$ incidence of TB within the first month of admission, with bacteriological confirmation in $38 \%$ of cases ( $n$ $201=8 / 21)$.

Children with HIV often present with paucibacillary TB disease that defies culturing, sample acquisition is difficult (particularly in the case of extrapulmonary TB) and malnutrition is a component of most clinical diagnostic scoring systems, rendering these less reliable in a population of malnourished children. ${ }^{22}$ We found few associations between routine laboratory parameters and culture-proven TB. Ultimately our best regression model was still not a particularly good fit for the data, likely due to unmeasured confounders and a lack of clinical and radiological details, and the associations we identified were not strong enough to be useful in a predictive model. Further studies are required to identify biomarkers of active TB that are reliable in severely-malnourished, HIV-infected children. ${ }^{23}$

In the regression model, sputum sampling was associated with a diagnosis of TB when compared with gastric aspirates, which is consistent with previous studies showing superior sensitivity of sputum sampling. ${ }^{24}$ Some of the other associations that we identified require a nuanced interpretation. The association of delayed ART and TB was seen in the fully adjusted model but not the final parsimonious model, and is not likely to be a true association, as all but one positive sputum sample were acquired during the first week of admission-i.e. early ART would not have prevented these diagnoses of TB. In addition, randomisation was stratified by TB status at enrolment. Possibly this finding indicates sampling bias by clinicians in this open-label study. The strongest conclusion one could draw is that the lack of increased risk of TB following early ART assuages concerns regarding immune reconstitution inflammatory syndrome (IRIS) reactions in these children.

Culture-proven bacterial infection was associated with a $14.4 \%$ reduced risk of cultureproven TB-not only is it exceedingly unlikely that bacterial infections are protective against mycobacterial infections, but multiple simultaneous opportunistic infections are in fact expected in severely immunosuppressed children. It is more likely that treatment for bacterial infections led to false-negative TB samples, or that treatment for TB resulted in 
false-negative bacterial cultures. Broad-spectrum antibacterials-empiric or otherwisewere frequently administered to the children in this study, as SAM is frequently associated with infections at time of presentation, and hospital-acquired infections are also common in this patient group. ${ }^{21}$ While the local formulary dictates that quinolones only be prescribed if indicated by antibiotic susceptibility profiles or on the advice of the infectious diseases service, aminoglycosides are frequently employed (benzylpenicillin and gentamicin are firstline empiric therapy for severely unwell children in this institution), as were carbepenems, ${ }^{21}$ both of which have activity against TB. ${ }^{25}$ We were unable to access the original charts for most patients, meaning that we could not correlate actual antibiotic prescriptions with the likelihood of a TB diagnosis. However, it is reasonable to assume that TB diagnoses could be masked by the treatment of other infections (or vice versa) in severely unwell children with HIV and SAM, or not considered when a child with SAM presents to hospital with a sepsislike syndrome and therefore it would be advisable to include TB investigation as part of the standard admission workup in order to minimize the risk of false negatives in the acute phase of malnutrition management.

This is, to our knowledge, the first study describing TB in children who all have both HIV and SAM. Looking beyond the multivariate model in a subset of patients, there were 13 additional patients in this study who were empirically treated for TB. The true rate of TB in children with SAM and HIV remains unquantifiable as long as so many cases remain unconfirmed. Table 3 summarises a sample of studies of such populations ${ }^{26,27}$ : while the rate of confirmed TB in children with SAM appears to rise in parallel with increasing rates of HIV (allowing for local variation in baseline TB rates), rates of empiric treatment vary substantially. Thus, it is impossible to assess the separate contributions of HIV and SAM to the pathogenesis of TB in children, though Table 3 does suggest that HIV is a key risk factor for TB in SAM- with increasing numbers of malnourished children now having HIV as an underlying cause, it is important for clinicians to particularly consider TB in such cases. Our own study shows that, despite the inherent challenges, microbiological confirmation of suspected pulmonary TB is possible in severely malnourished children with HIV.

Other studies in Africa have started with a diagnosis of TB and looked backwards for associations with malnutrition and/or HIV, usually finding strong and independent associations with both of these risk factors and TB mortality. ${ }^{12,13,28}$ The only other studies 
258

259

260

261

262

263

264

265

266

267

268

269

270

271

272

273

274

275

276

277

278

279

280 that we can find that began with malnourished children and looked for associations with TB were carried out in South Asia, where paediatric HIV prevalence is far lower, and differ in many important methodological factors from our study. ${ }^{29,30}$ One such study examined 405 severely malnourished Bangladeshi children with respiratory symptoms and radiographic pulmonary infiltrates: $7 \%$ had confirmed TB and a further $16 \%$ were treated based on clinical suspicion. ${ }^{30}$ HIV prevalence was not determined, but was known to be rare in that locale. While this study is important in raising awareness of TB mimicking acute pneumonia in children with SAM, the TB prevalence in a select population with radiologic changes will obviously be higher than the TB prevalence in malnourished children in general.

Strengths of our study include prospective recruitment of patients and access to tertiarylevel diagnostic facilities. Limitations include the relatively small number of patients, missing data, practice changes during the course of the trial (eg. the introduction of Xpert) and the single-centre setting, limiting the generalisability to other settings in sub-Saharan Africa and beyond. Poor availability of patients' original hospital files meant that we lacked data regarding clinical findings, radiographs and tuberculin skin tests, which would have been a useful addition to our analysis. We considered our two patients who were smearpositive but culture negative to have confirmed TB, in keeping with the WHO classification, ${ }^{20}$ but these may have been false positives due to non-tuberculous mycobacteria-equally, we considered patients who started treatment in other centres to be empirically-treated, when they may in fact have had a confirmed diagnosis which was not identifiable on the national laboratory online system. 


\section{CONCLUSIONS}

282 TB is very common in HIV-infected children with severe acute malnutrition. While

283 microbiological confirmation of the diagnosis is feasible, numerous diagnostic challenges-

284 including suboptimal testing (gastric aspirates as opposed to induced sputum) and false

285 negative TB diagnostics (with prior antibacterial therapy)-mean that empiric treatment

286 remains common in this patient group. Future studies should focus on diagnostic strategies

287 that are sufficiently robust for this important, vulnerable population of children in resource-

288 poor settings. With SAM being increasingly seen in conjunction with HIV in sub-Saharan

289 Africa, it is important that TB diagnosis and treatment be integrated into the programmatic management of these conditions.

291

292 
293 ACKNOWLEDGEMENTS

294 We thank all of the participants and their carers. In addition, we are grateful to the 295 members of the LivTB research group in Liverpool for helpful discussions and to Ms 296 Thobekile Sibaya for administrative support.

297 
1. Van Lettow M, Fawzi WW, Semba RD. Triple trouble: the role of malnutrition in tuberculosis and human immunodeficiency virus co-infection. Nutr Rev. 2003;61(3):81-90.

6. Black RE, Victora CG, Walker SP, Bhutta ZA., Christian P, De Onis M, et al. Maternal and child undernutrition and overweight in low-income and middle-income countries. Lancet. 2013;382(9890):427-51.

7. UNAIDS. Global report: UNAIDS report on the global AIDS epidemic 2012 [Internet]. Available from: http://www.unaids.org/sites/default/files/media_asset/20121120_UNAIDS_Global_R eport_2012_with_annexes_en_1.pdf

8. World Health Organisation. World Health Organisation Country Profile: South Africa [Internet]. [accessed Sep 3 2016]. Available from: http://www.who.int/countries/zaf/en/

9. Cobb G, Bland RM. Nutritional supplementation: The additional costs of managing children infected with HIV in resource-constrained settings. Trop Med Int Heal. 2013;18(1):45-52.

10. Heikens GT, Bunn J, Amadi B, Manary M, Chhagan M, Berkley JA, et al. Case management of HIV-infected severely malnourished children: challenges in the area of highest prevalence. Lancet. 2008;371:1305-7.

11. Chinkhumba J, Tomkins A, Banda T, Mkangama C, Fergusson P. The impact of HIV on mortality during in-patient rehabilitation of severely malnourished children in Malawi. Trans R Soc Trop Med Hyg. 2008;102:639-44. 
12. Hicks RM, Padayatchi N, Shah NS, Wolf A, Werner L, Sunkari VB, et al. Malnutrition associated with unfavorable outcome and death among South African MDR-TB and HIV co-infected children. Int J Tuberc Lung Dis. 2014;18(9):1074-83.

13. Hesseling AC, Westra a E, Werschkull H, Donald PR, Beyers N, Hussey GD, et al. Outcome of HIV infected children with culture confirmed tuberculosis. Arch Dis Child. 2005;90:1171-4.

14. Cegielski JP, McMurray DN. The relationship between malnutrition and tuberculosis: Evidence from studies in humans and experimental animals. Int J Tuberc Lung Dis. 2004;8(August 2003):286-98.

15. Jaganath $D$, Mupere E. Childhood tuberculosis and malnutrition. J Infect Dis. 2012;206(12):1809-15.

16. Cotton MF, Schaaf HS, Hesseling AC, Madhi SA. Childhood TB HIV and childhood tuberculosis : the way forward. Int J Tuberc Lung Dis. 2004;8(5):675-82.

17. Archary $M$, Bobat $R$, Larussa P. Severe acute malnutrition and timing of anti-retroviral initiation in children with HIV. International AIDS Society. 2014. p. MOPE066.

18. World Health Organisation. WHO child growth standards and the identification of severe acute malnutrition in infants and children. Geneva: World Health Organization, United Nations Children's Fund; 2009.

19. World Health Organisation. Guideline: Updates on the management of severe acute malnutrition in infants and children. Geneva: World Health Organisation; 2013.

20. World Health Organisation. Definitions and reporting framework for tuberculosis 2013 revision [Internet]. 2013. Available from: http://apps.who.int/iris/bitstream/10665/79199/1/9789241505345_eng.pdf

21. Archary M, Adler H, La Russa P, Mahabeer P, Bobat RA. Bacterial infections in HIVinfected children admitted with severe acute malnutrition in Durban, South Africa. Paediatr Int Child Health [Internet]. 2016;1-8. Available from: http://www.tandfonline.com/doi/full/10.1080/20469047.2016.1198561

22. Jones KDJ, Berkley JA. Severe Acute Malnutrition and Infection. 2014;1-29.

23. Kroidl I, Clowes P, Reither K, Mtafya B, Rojas-Ponce G, Ntinginya EN, et al. Performance of urine lipoarabinomannan assays for paediatric tuberculosis in Tanzania. Eur Respir J. 2015;46(3):761-70.

24. Zar HJ, Hanslo D, Apolles P, Swingler G, Hussey G. Induced sputum versus gastric lavage for microbiological confirmation of pulmonary tuberculosis in infants and young children: A prospective study. Lancet. 2005;365(9454):130-4. 
25. Diacon $A H$, van der Merwe $L$, Barnard $M$, von Groote-Bidlingmaier $F$, Lange $C$, GarcíaBasteiro AL, et al. $\beta$-Lactams against Tuberculosis - New Trick for an Old Dog? N Engl J Med 2016;NEJMc1513236. Available from:

http://www.nejm.org/doi/10.1056/NEJMc1513236

26. Cartmell E, Natalal H, François I, Ferreira MH, Grahnquist L. Nutritional and clinical status of children admitted to the malnutrition ward, Maputo Central Hospital: A comparison of data from 2001 and 1983. J Trop Pediatr. 2005;51(2):102-5.

27. De Maayer T, Saloojee H. Clinical outcomes of severe malnutrition in a high tuberculosis and HIV setting. Arch Dis Child. 2011;96:560-4.

28. Soeters M, de Vries AM, Kimpen JLL, Donald PR, Schaaf HS. Clinical features and outcome in children admitted to a TB hospital in the Western Cape--the influence of HIV infection and drug resistance. S Afr Med J. 2005;95(8):602-6.

29. Bhat PG, Kumar AM V, Naik B, Satyanarayana S, Deepak KG, Nair SA., et al. Intensified tuberculosis case finding among malnourished children in nutritional rehabilitation centres of Karnataka, India: Missed opportunities. PLoS One. 2013;8(12):8-14.

30. Chisti MJ, Graham SM, Duke T, Ahmed T, Ashraf H, Faruque ASG, et al. A prospective study of the prevalence of tuberculosis and bacteraemia in Bangladeshi children with severe malnutrition and pneumonia including an evaluation of Xpert MTB/RIF assay. PLoS One. 2014;9(4). 

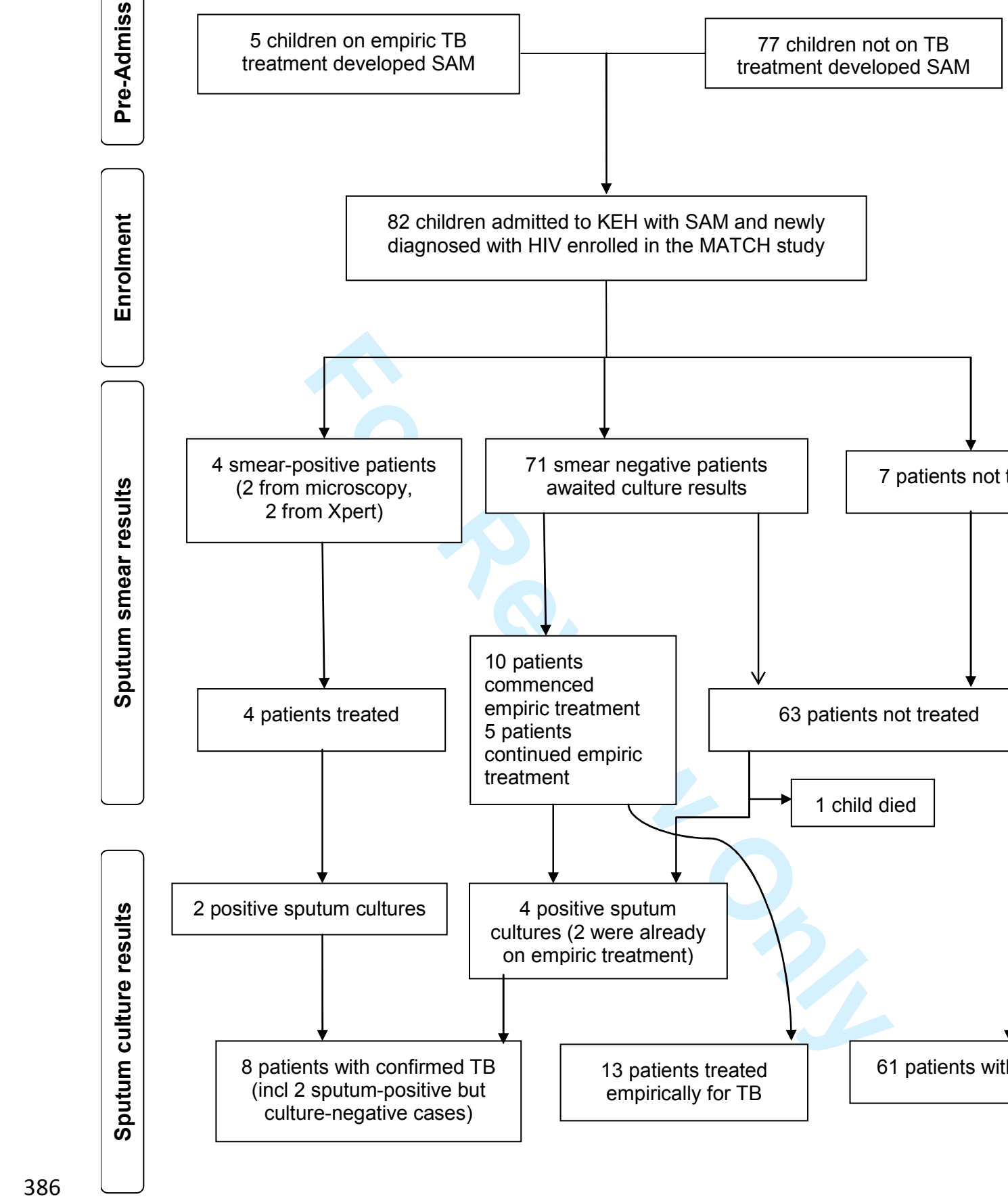

82 children admitted to KEH with SAM and newly diagnosed with HIV enrolled in the MATCH study
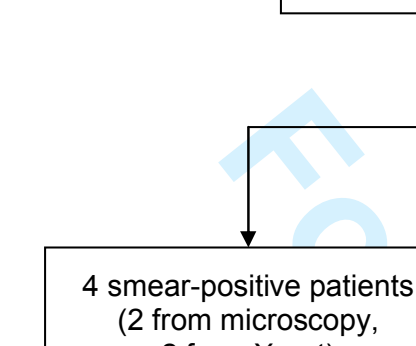
2 from Xpert)
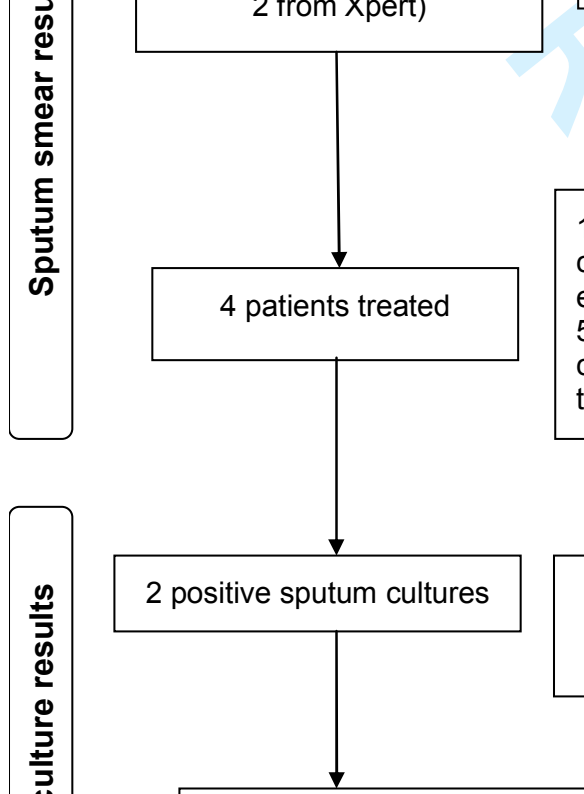

8 patients with confirmed TB (incl 2 sputum-positive but culture-negative cases)

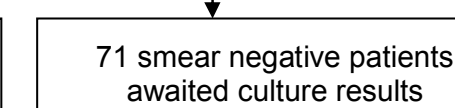

Figure 1: Recruitment and diagnostic process 


\begin{tabular}{|c|c|c|c|c|}
\hline & $\begin{array}{l}\text { TB negative }(n= \\
61)\end{array}$ & $\begin{array}{l}\text { Bacteriologically } \\
\text { confirmed }(n=8)\end{array}$ & $\begin{array}{l}\text { Clinically diagnosed } \\
(n=13)\end{array}$ & $p$ \\
\hline \multicolumn{5}{|l|}{ BASELINE CHARACTERISTICS } \\
\hline Age (months) & $19 \pm 22$ & $29 \pm 41$ & $36 \pm 40$ & 0.1 \\
\hline Female & $28(45.9 \%)$ & $5(62.5 \%)$ & $5(38.5 \%)$ & 0.56 \\
\hline Oedema present & $12(19.7 \%)$ & $4(50 \%)$ & $2(15.4 \%)$ & 0.12 \\
\hline Early ART (<2 weeks) & $29(47.5 \%)$ & $3(37.5 \%)$ & $6(46.2 \%)$ & 0.87 \\
\hline Other bacterial infection & $33(54.1 \%)$ & $3(37.5 \%)$ & $3(23.1 \%)$ & 0.1 \\
\hline CD4 (cells $/ \mu L)(n=73)$ & $945 \pm 807$ & $874 \pm 309$ & $721 \pm 214$ & 0.49 \\
\hline CD4 percentage $(n=73)$ & $18.35 \pm 9.1 \%$ & $15.5 \pm 9.83 \%$ & $14.5 \pm 11.4 \%$ & 0.4 \\
\hline Haemoglobin $(\mathrm{g} / \mathrm{dL})$ & $8.65 \pm 2.0$ & $8.7 \pm 3.8$ & $8.4 \pm 1.7$ & 0.73 \\
\hline Haemoglobin $<7$ & $9(14.8 \%)$ & $3(37.5 \%)$ & $2(15.4 \%)$ & 0.27 \\
\hline Platelets (x 10\% /L) & $336 \pm 208$ & $336 \pm 215$ & $297 \pm 131$ & 0.81 \\
\hline Platelets $>400$ & $20(32.8 \%)$ & $1(12.5 \%)$ & $4(30.8 \%)$ & 0.6 \\
\hline Albumin (g/L) & $24 \pm 8$ & $21 \pm 5$ & $23 \pm 6$ & 0.56 \\
\hline Albumin $<25$ & $30(29.2 \%)$ & $6(75 \%)$ & $8(61.5 \%)$ & 0.35 \\
\hline CRP $(\mathrm{mg} / \mathrm{L})(\mathrm{n}=37)$ & $38 \pm 49$ & $38 \pm 41$ & $57 \pm 35$ & 0.68 \\
\hline CRP $>10(n=37)$ & 16 & 5 & 5 & 0.17 \\
\hline \multicolumn{5}{|l|}{ SITE OF TB } \\
\hline Pulmonary & $\sqrt{2}$ & 7 & 8 & - \\
\hline Pulmonary + Disseminated & +1 & 1 & 3 & - \\
\hline Meningitis & - & 0 & 1 & - \\
\hline Abdominal & - & 0 & 1 & - \\
\hline \multicolumn{5}{|l|}{ DIAGNOSIS } \\
\hline Smear microscopy & - & $2^{*}-2=$ & - & - \\
\hline Xpert & - & $2+$ & - & - \\
\hline Culture & - & 6 & - & - \\
\hline
\end{tabular}

389 Table 1: Baseline characteristics of the patients in this study.

390 Values are given as mean \pm SD or $\mathrm{n}(\%)$ as appropriate. $\mathrm{P}$ values are for a three-way 391 comparison. Bacterial infection refers to any positive blood/sputum/urine culture for 392 pathogenic bacteria within the first month of admission.

$393 *$ Both were smear positive but culture negative

394 †Both were culture positive 
Page 19 of 22

398
399

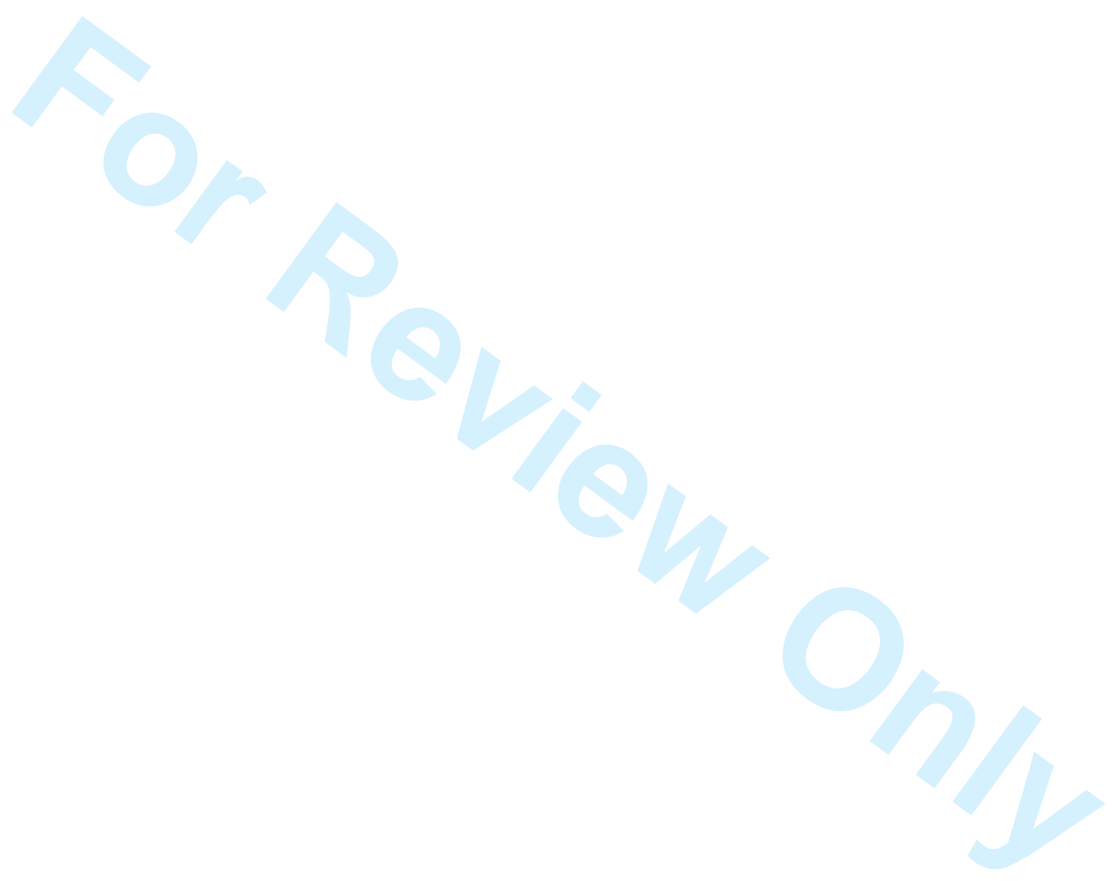




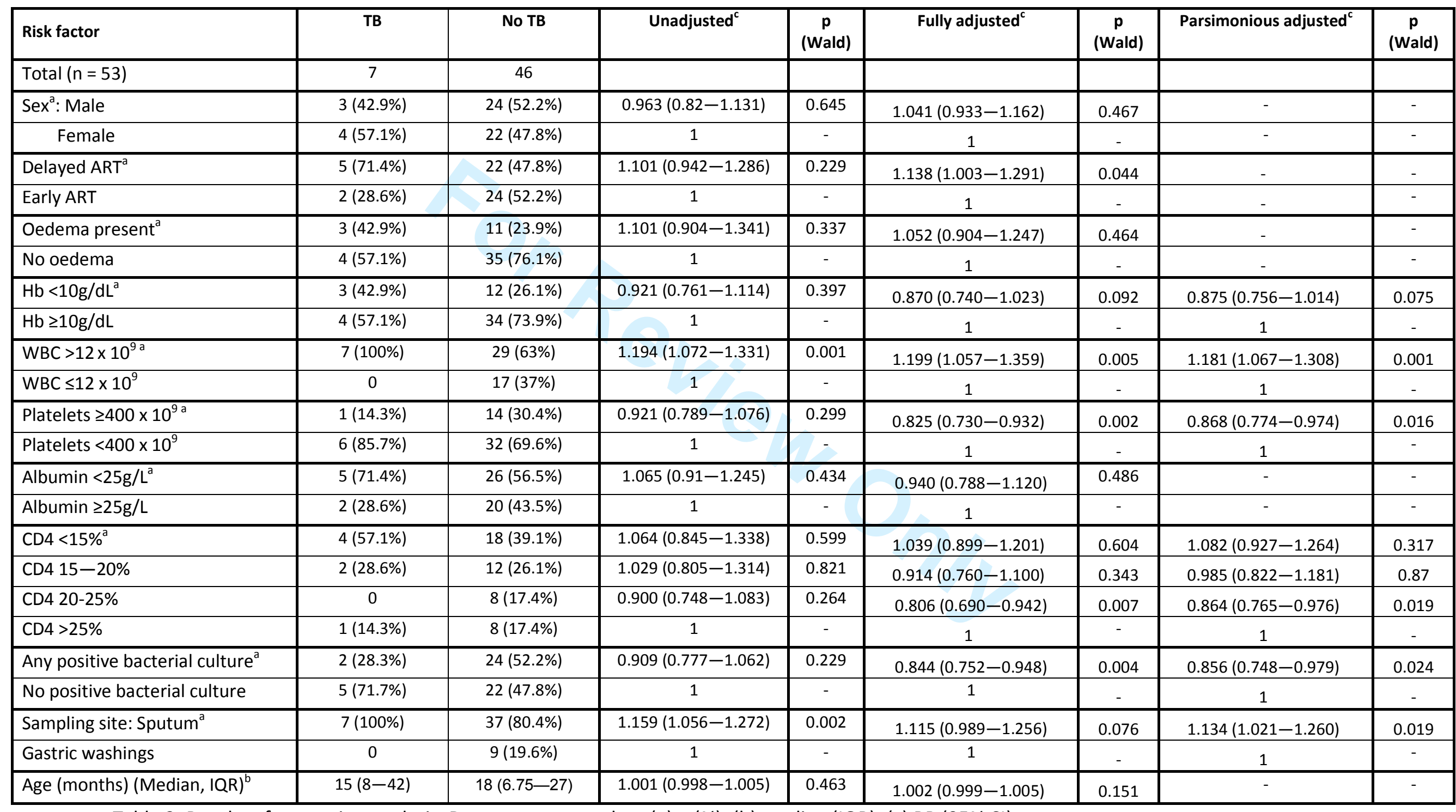

400 Table 2: Results of regression analysis. Data are presented as: (a) $n$ (\%); (b) median (IQR); (c) RR (95\% CI) 


\begin{tabular}{|c|c|c|c|c|c|c|c|}
\hline Study & Year & Setting & $\begin{array}{l}\text { Number } \\
\text { with SAM }\end{array}$ & $\begin{array}{l}\text { Prevalence } \\
\text { of HIV }^{\mathrm{a}}\end{array}$ & $\begin{array}{l}\text { Prevalence of } \\
\text { confirmed } T^{a}\end{array}$ & $\begin{array}{l}\text { Prevalence of empiric } \\
\text { TB treatment }{ }^{\mathrm{a}}\end{array}$ & $\begin{array}{l}\text { Overall rate of TB } \\
\text { treatment }^{\mathrm{a}}\end{array}$ \\
\hline Cartmell (Part 1) & 1983 & $\begin{array}{l}\text { Maputo, } \\
\text { Mozambique }\end{array}$ & 833 & 0 & $53(6.4 \%)$ & $29(3.5 \%)$ & $81(9.7 \%)$ \\
\hline Cartmell (Part 2) & 2001 & $\begin{array}{l}\text { Maputo, } \\
\text { Mozambique }\end{array}$ & 558 & $65(29.3 \%)^{*}$ & $78(14 \%)$ & $14(2.5 \%)$ & $92(16.5 \%)$ \\
\hline De Maayer $^{27}$ & 2011 & $\begin{array}{l}\text { Johannesburg, } \\
\text { South Africa }\end{array}$ & 113 & $58(51 \%)$ & $3(2.6 \%)$ & $29(25.7 \%)$ & $32(28.3 \%)$ \\
\hline This study & 2016 & $\begin{array}{l}\text { Durban, South } \\
\text { Africa }\end{array}$ & 82 & $82(100 \%)$ & $8(9.8 \%)$ & $13(15.9 \%)$ & $21(25.6 \%)$ \\
\hline
\end{tabular}

401 Table 3: Examples of other studies of children admitted for the management of SAM in different sub-Saharan African settings. These data suggest that, as 402 rates of HIV increase, so too do rates of bacteriologically confirmed TB in children with SAM-however, rates of empiric treatment differ markedly between 403 studies and confound accurate assessment.

$404 \quad$ (a) $n(\%)$

$405 * 51$ with an AIDS-defining-illness, 14 with confirmed HIV. An additional 30 had suspected HIV but this was not confirmed (not included in this table) 


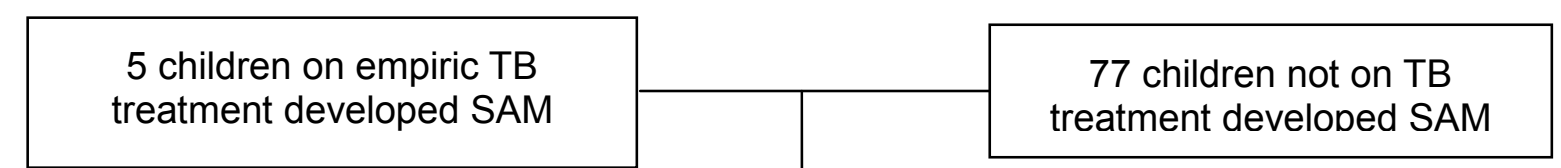
diagnosed with HIV enrolled in the MATCH study
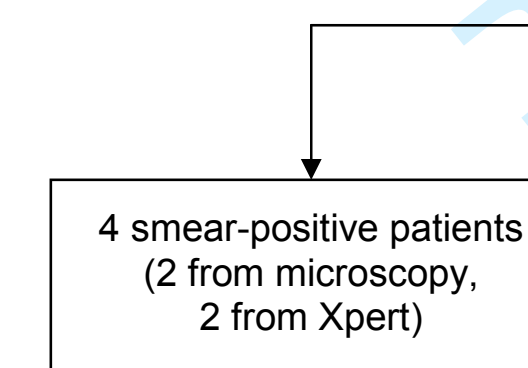

4 patients treated

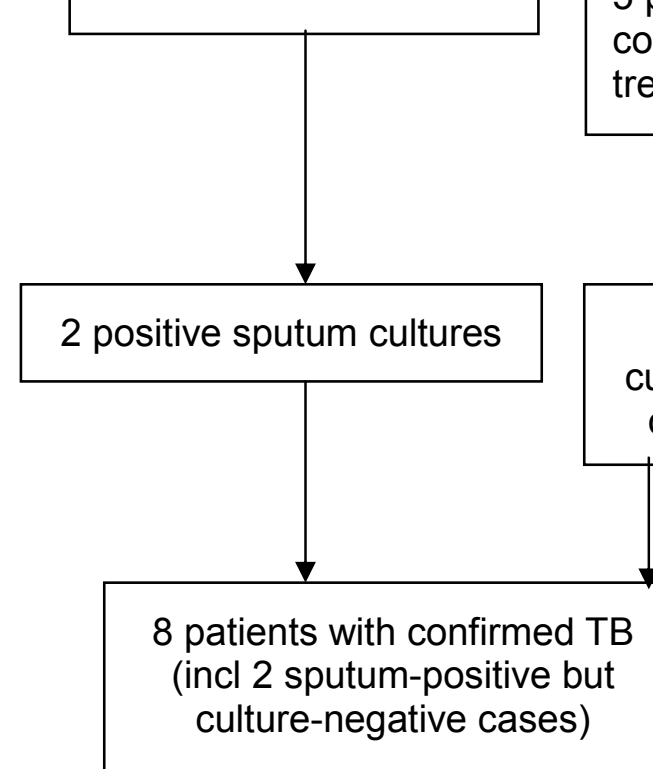

71 smear negative patients awaited culture results

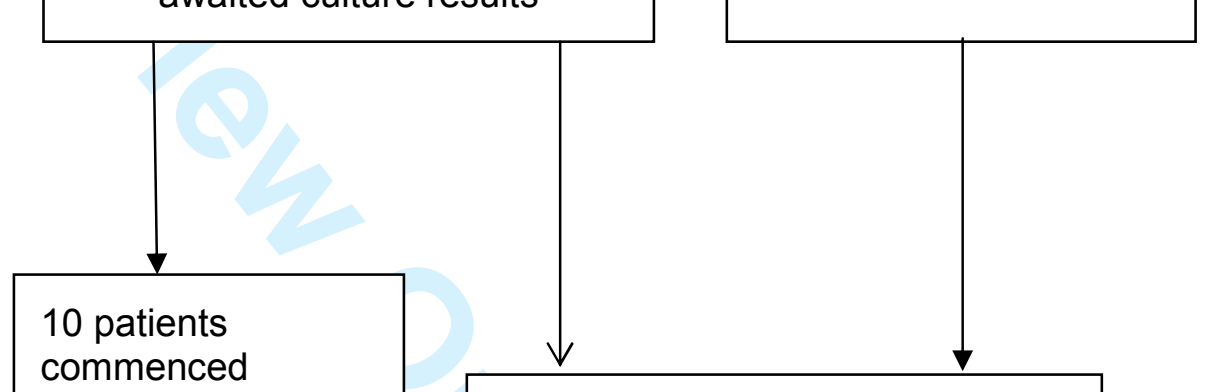

empiric treatment 5 patients continued empiric treatment 